\title{
Desarrollo de las competencias específicas relacionadas con la tecnología para las áreas de periodismo y comunicación audiovisual
}

\author{
Antonio J. Benítez IgLEsiAs \\ Universidad Carlos III de Madrid \\ abenitez@hum.uc3m.es \\ Esteban M. STEPANIAN TARACIDO \\ Universidad Carlos III de Madrid \\ estepani@hum.uc3m.es
}

Recibido: 27/07/2012

Aceptado: 31/10/2012

\begin{abstract}
Resumen
El cambio hacia los estándares formativos que moviliza el EEES constituye una oportunidad para mejorar el enfoque de la capacitación de los estudiantes de las áreas de Comunicación para el ejercicio profesional. Esta adaptación afronta algunos retos complicados, como son el necesario uso de las tecnologías audiovisuales o los recursos limitados para aplicarla. Se propone una metodología combinada, que reúne elementos positivos de la autoformación, del aprendizaje en entornos colaborativos, del uso de las TIC y del trabajo en equipo. Los alumnos participantes han contestado a un cuestionario que les pregunta sobre el papel de la tecnología audiovisual en su formación y en el entorno profesional y han realizado vídeos didácticos para ser utilizados por sus compañeros en la mejora de las capacidades de uso de la tecnología.
\end{abstract}

Palabras clave: Tecnología Audiovisual; Periodismo y Comunicación Audiovisual; Aprendizaje; Universidad.

\section{Developing of specific technological skills related in Journalism and Media Production}

\begin{abstract}
Makings changes to learning standards that are suggested by EHEA are a good opportunity to enhance students' professional competences about Journalism and Media Production. This approach involves difficult challenges, like skills in audiovisual technologies and limited resources to apply it. A combined methodology is proposed, putting together positive elements from self-learning, from collaboration environment, from the use of IT and from team work. Participating students have answered a survey asking them about the audiovisual technology role in their training and in the professional environment, and have produced some didactic videos their fellows could see to improve their skills in the use of technology.
\end{abstract}

Keywords: Audiovisual Technology; Journalism and Media Practices; Learning; University

\section{Referencia normalizada}

BENÍTEZ IGLESIAS, Antonio J. y STEPANIAN TARACIDO, Esteban M. (2012): "Desarrollo de las competencias específicas relacionadas con la tecnología para las áreas de periodismo y comunicación audiovisual". Estudios sobre el mensaje periodístico. Vol. 18, núm. especial octubre, págs.: 129-140. Madrid, Servicio de Publicaciones de la Universidad Complutense.

Sumario: 1. Introducción. Fuentes y estado de la cuestión; 1.1. Consideraciones pedagógicas. 2. Metodología. 3. Desarrollo. Resultados. 4. Conclusiones. 5. Referencias bibliográficas. 


\section{Introducción. Fuentes y estado de la cuestión}

El constante desarrollo y la creciente complejidad de uso de las tecnologías de la información han colocado a los estudios superiores del área de Periodismo y Comunicación Audiovisual en un problema de difícil solución. La formación integral del periodista de radio o televisión, o la de los productores o realizadores audiovisuales requiere, como condición previa, un cierto grado de conocimiento y competencia en el manejo de diferentes dispositivos y softwares de producción.

Inicialmente este requisito no debería causar sorpresa. Es natural que a los alumnos de las asignaturas relacionadas con el área de Lingüística y Literatura se les presuponga la competencia en el uso de uno o más procesadores de texto o a los alumnos del área de Biología se les forme en el conocimiento y uso del microscopio y sus programas de software asociados.

Sin embargo, el volumen y diversidad de competencias relacionadas con las tecnologías de la información provocan que una amplia mayoría del alumnado presente dificultades y carencias de operación, absolutamente explicables en el actual contexto y, en muchas ocasiones, extensibles a parte del profesorado no específico. Producir un programa informativo en un plató de televisión, por poner un ejemplo inmediato, requiere el conocimiento de una serie de técnicas y operativas específicas ajenas a los contenidos de asignaturas como "Periodismo televisivo" o "Los informativos en televisión" y probablemente ajenas también a la capacitación de su profesor responsable.

A menudo las soluciones a este problema tienen un carácter extremo. O bien, en la medida de lo posible, se obvian estos inconvenientes imponiendo a los alumnos unas rutinas de trabajo absolutamente infundadas desde el punto de vista conceptual: "cuando este indicador rojo se ilumine debe oprimirse este botón". O bien, en el otro extremo de la balanza, se termina por reducir los programas y contenidos de las asignaturas a simples sesiones de capacitación del alumnado en el manejo de uso de determinado dispositivo o software informático confundiendo así, poniendo por caso, la teoría de la edición audiovisual con el manual de uso de Final Cut o Avid, o la discusión sobre la evolución en composición de la imagen con la capacidad de manejar Photoshop.

La primera de las soluciones enumeradas supondría provocar frustración en un alumnado tendente a identificar sus limitaciones tecnológicas con carencias intelectuales. En la segunda se reducirían los estudios superiores a una escuela de capacitación tecnológica con contenidos más propios de otros ámbitos educativos.

Estas dos opciones extremas no hacen más que poner de manifiesto los problemas que quizá en menor medida presentan también las consideradas vías intermedias.

Como puede comprobarse en el Libro Blanco de Títulos de Grado en Comunicación, en las áreas de Periodismo y de Comunicación Audiovisual, se han diseñado los planes de estudio que fomentan la implantación del Espacio Europeo de Educación Superior (EEES) con el objetivo de atender a "un aumento de la necesidad de profesionales cualificados en el campo de la Comunicación" (ANECA, 2005: 16).

En el mismo documento se expone el modelo de estudios escogido, que establece como una de las exigencias de formación para el Título de Periodismo la "experimentación derivada de la práctica en laboratorios y profesional”. Para alcanzar sus 
objetivos, el alumno participante en este Grado debería adquirir conocimientos y capacidades que incluyesen, entre otros, aspectos de formación en el "conocimiento y uso de la tecnología (informativa e informática) que permita elaborar productos periodísticos en cualquier soporte y sistema" (ANECA, 2005: 309).

Con respecto al Grado en Comunicación Audiovisual, el documento de la Agencia hace especial mención, entre los objetivos fundamentales, a los recursos tecnológicos (ANECA, 2005: 320) y resalta, entre los aspectos formativos principales, además de las capacidades expresivas y "los lenguajes de cada uno de los medios y formatos de la comunicación", el conocimiento y uso de las tecnologías de la comunicación (ANECA, 2005: 321). En la estructura general de ambos Grados que se propone, se reservan 16 créditos para las Tecnologías Informativas entre los contenidos instrumentales obligatorios, con un total de horas recomendadas entre 400 a 480 y con un porcentaje de práctica del 45\% (ANECA, 2005: 317-318 y 325). En la estructura del Grado en Comunicación Audiovisual, en concreto, las Tecnologías de los Medios Audiovisuales y Multimedia están contempladas entre los contenidos comunes obligatorios, y a las técnicas se les reservan, además, 30 créditos (entre 750 y 900 horas de trabajo del estudiante, (ANECA, 2005: 327)

Así, se pretende que los alumnos conozcan y manejen una serie de herramientas cuyo uso razonable exige una inversión de tiempo en todo caso superior al número de horas que la propia distribución docente (Sánchez et al., 2011: 660) finalmente puede dedicar a las asignaturas implicadas.

Si bien algunos autores afirman que los profesores de materias relacionadas con radio y televisión ya venían utilizando anteriormente metodologías combinadas cercanas a las que ahora se recomiendan, y en este sentido "se sitúan en una posición aventajada para la implantación del EEES" (Jiménez y Moncholi, 2009: 147), lo cierto es que, para superar el reto en las actuales circunstancias, demasiado a menudo se ven abocados a degradar los objetivos de la educación superior o, en el mejor de los casos, a fomentar una indeseable mecanización inconsciente de técnicas y rutinas de trabajo de las que el alumno debería conocer tanto su trasfondo teórico como sus fundamentos.

Cabe preguntarse entonces, y esta es la hipótesis de partida de este trabajo, si sería posible adoptar una tercera vía de solución, valiéndose de recursos educativos alternativos a la docencia presencial, que permitiese al alumnado adquirir previa, o paralelamente, las rutinas de trabajo y la capacitación tecnológica específica necesaria para llevar a buen fin los objetivos docentes de las asignaturas implicadas, evitando así los problemas de interferencia antes descritos.

\subsection{Consideraciones pedagógicas}

En su Tratado de pedagogía, Kant planteaba ya en 1803 los beneficios del aprendizaje autónomo y actualmente, doscientos años después, nadie parece dudar, de la eficacia de las técnicas de autoaprendizaje en todos los ámbitos y niveles de la educación. Desde diferentes puntos de vista, numerosos autores han insistido en ello: Tough, Knowles, Long, Aebli, Dumazedier, el grupo Graf... (Carré, 2006: 6).

"La persona que es capaz de aprender de forma autónoma sabe lo que quiere; qué tiene que hacer o aprender para obtenerlo en entornos que cambian con rapidez y exi- 
gen flexibilidad y adaptabilidad; se enfrenta de modo crítico con los datos obtenidos; amplía o profundiza la investigación si es necesario y asume un mayor control sobre su propio proceso de aprendizaje, autorregulándolo" (Ruiz 2004: 48).

En sintonía, Joan Rué considera que fomentar las capacidades de autoformación en el alumnado debe constituir uno de los objetivos prioritarios de la educación superior y alcanzarlo debe de ser considerado, sin duda alguna, como uno de los mayores éxitos a los que esta puede aspirar (Rué, 2009: 82).

En este contexto son también múltiples los estudios que ensalzan los entornos de estudio colaborativo en los que los alumnos integrados en un grupo de trabajo intercambian experiencias y transmiten los conocimientos adquiridos a sus propios compañeros o estudiantes de cursos inferiores.

Las diversas investigaciones que desde hace ya tiempo vienen desarrollando entidades tan acreditadas como el NTL Institute for Applied Behavioral Science acerca de diferentes e innovadoras técnicas docentes confirman que "enseñar a otro" es una práctica muy eficiente para el aprendizaje en términos de retención cognitiva de los contenidos (Wood, 2004: 4). Aunque, como también ha puesto de manifiesto David Boud (2001: 170 y ss.), la eficacia del aprendizaje peer to peer ${ }^{1}$ es patente únicamente cuando este se adecua a los rigurosos límites y normas que debe imponer el profesor al diseñar estrategias de blended learning ${ }^{2}$ específicas para cada uno de los problemas concretos que se puedan plantear.

Los estudios e investigaciones llevados a cabo por la prestigiosa John Hopkins University confirman también la potencia didáctica de las experiencias en la que se invita a los alumnos a participar en la formación de sus compañeros incitándoles a utilizar sus propias técnicas de entretenimiento audiovisual (Singhal y Brown, 1996). La responsabilidad de preparar recursos didácticos por parte de los estudiantes para sus iguales incide en el grado de compromiso (Cenich y Santos, 2005: 4) y de profundización sobre los temas seleccionados, además de adecuar en gran medida el tratamiento empleado a las expectativas de sus pares.

Los estudiantes implicados cambian por lo tanto su rol de "consumidores de información" al de "generadores de conocimiento", reforzando su sentimiento de pertenencia a la red de aprendizaje colaborativo. La posibilidad de que el alumno pase de consumidor a generador de conocimiento es para Ruiz (2004: 47) uno de los mayores desafíos de la actual labor docente.

El estudiante debe servirse de las tecnologías de la información para crear recursos docentes que mejoren acumulativamente su competencia técnica en el manejo de las mismas y, como consecuencia, esta le permita explotar al máximo sus posibilidades expresivas. Esta peculiaridad del uso de las tecnologías de la información en el área de conocimiento de la Comunicación Audiovisual es un caso paradigmático de

${ }^{1}$ Peer to peer: o aprendizaje inter pares se dice de la estrategia de enseñanza-aprendizaje en la que los compañeros del mismo nivel de conocimientos se intercambian conocimientos o experiencias formativas entre sí.

2 Blended learning: concepción del método formativo que consiste en mezclar elementos de la metodología presencial con otros de enseñanza a distancia. 
la unión de las cuatro ventajas fundamentales que, a juicio de Wang y Kinuthia (2004: 3), proveen las Tecnologías de la Información y de la comunicación (TIC) en ámbitos educativos: "A technology enhanced learning environment enjoys following characteristics: (1) using technology to motivate people, (2) using technology to enrich learning resources, (3) using technology to implement learning and instructional strategies, and (4) using technology to assess and evaluate learning goals".

El uso de vídeos formativos en el entorno de las TIC como recursos de apoyo a la docencia en ámbitos de blended learning ha sido profusamente defendido tanto desde el punto de vista teórico general (Aebli, 2001; Rué 2009; Ruiz, 2004) como en su aplicación a algunos ámbitos concretos de aprendizaje (Aparicio, 1985; Bravo, 1992; Cabero y Márquez, 2002; Díaz y Noriega, 2009). El lenguaje audiovisual es el más eficaz cuando se trata de mostrar y explicar procedimientos cuya descripción textual exige tediosas y confusas descripciones. Aprender a efectuar un balance de blancos en una cámara de vídeo o disponer los envíos auxiliares en una mesa de mezclas de sonido son ejemplos obvios en lo que respecta a operaciones sencillas pero la afirmación es válida también, incluso cuando se trata de exponer conceptos de mayor complejidad, que escapan del ámbito puramente tecnológico, para adentrarse en el de la Realización Audiovisual como pueden ser la construcción del espacio fílmico, el respeto al eje de acción o la composición multicapa.

La bibliografía consultada alienta a establecer una estrategia docente que faculte a los profesores de las áreas de Periodismo y Comunicación Audiovisual para diseñar métodos de autoaprendizaje basados en entornos colaborativos que proporcionen al alumnado, bajo supervisión y al margen del tiempo asignado a los contenidos centrales de la asignatura, la capacitación tecnológica que le permita realizar las tareas prácticas con suficiente solvencia.

\section{Metodología}

En una primera fase se solicitó a un grupo de profesores (siete) que desarrollan su actividad docente en el área de Comunicación Audiovisual que invitasen a sus alumnos a determinar el papel que consideran que su capacitación tecnológica debería ocupar durante sus estudios universitarios y el valor que, según su opinión, podría tener en un previsible futuro laboral. Se recogieron los datos a partir de un test de Likert propuesto en una encuesta en línea con una serie de preguntas. El análisis de los resultados, que no pretende adquirir valor universal, sí permite obtener las primeras impresiones sobre las demandas de los alumnos (casi doscientos contestaron los cuestionarios) y situar la discusión desde un punto de vista adecuado.

El diseño de la encuesta propuesta durante la primera fase del proyecto preveía obtener indicaciones sobre tres aspectos fundamentales. En primer lugar se pretendía valorar la preparación audiovisual del alumno mediante una serie de preguntas acerca de su experiencia como mero espectador de medios audiovisuales; además se intentaba estimar en qué grado los conocimientos previos presupuestos en las asignaturas implicadas habían sido realmente adquiridos y, por último, se trataba de indagar sobre las demandas formativas del estudiante en cuanto a su capacitación tecnológica y su percepción acerca de la exigencia de estas capacidades en los ámbitos profesionales. 
En la segunda fase, se ha propuesto como una de las actividades prácticas de la docencia (en cuatro asignaturas distintas), que a lo largo de un semestre, los alumnos, organizados en equipos de producción, elaborasen vídeos didácticos de aproximadamente un minuto de duración sobre un corpus de argumentos específicos de carácter tecnológico (más de doscientos diez ítems) que englobaban todos aquellos cuyo conocimiento se ha considerado imprescindible por los profesores del área (ocho en esta fase).

La realización de estos vídeos didácticos tiene un doble objetivo. Se trata, por un lado, de que los alumnos investiguen a fondo y de manera autónoma durante la preparación del guion el argumento elegido -mejorando así su competencia en él- y, por otro, que elaboren productos atractivos desde el punto de vista audiovisual. Casi doscientos diez estudiantes finalizaron sus vídeos, que después se incluyeron en un servidor o videoblog, de forma que estén a disposición de sus compañeros para mejorar su conocimiento de los dispositivos de producción y de las rutinas de trabajo precisas, transmitiendo así su conocimiento con la estrategia peer to peer.

En resumen, desde el punto de vista metodológico, la experiencia propuesta constituye un sencillo caso de demostración por construcción. La posibilidad de activar un sistema de aprendizaje colaborativo y autónomo que verifique eficazmente los requisitos impuestos por la hipótesis es condición suficiente para que esta se pueda considerar validada.

\section{Desarrollo. Resultados}

De entre los resultados obtenidos cabe destacar las respuestas a las preguntas 6, 8, 12, 13 y 15 , del cuestionario (ver gráficos) porque son especialmente reveladoras.

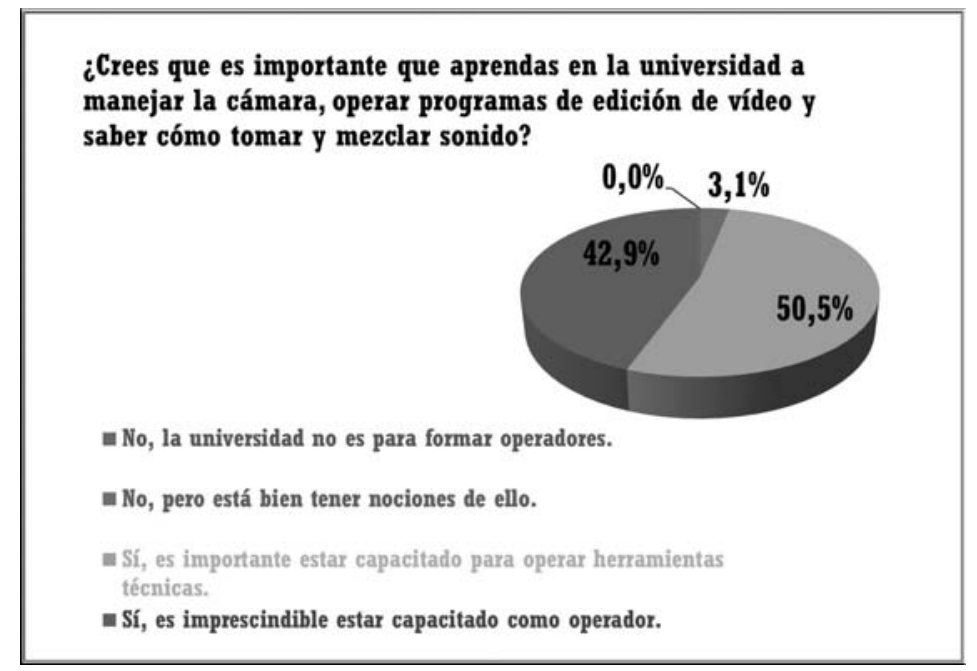

Gráfico 1. Pregunta 6. Fuente: elaboración propia. 


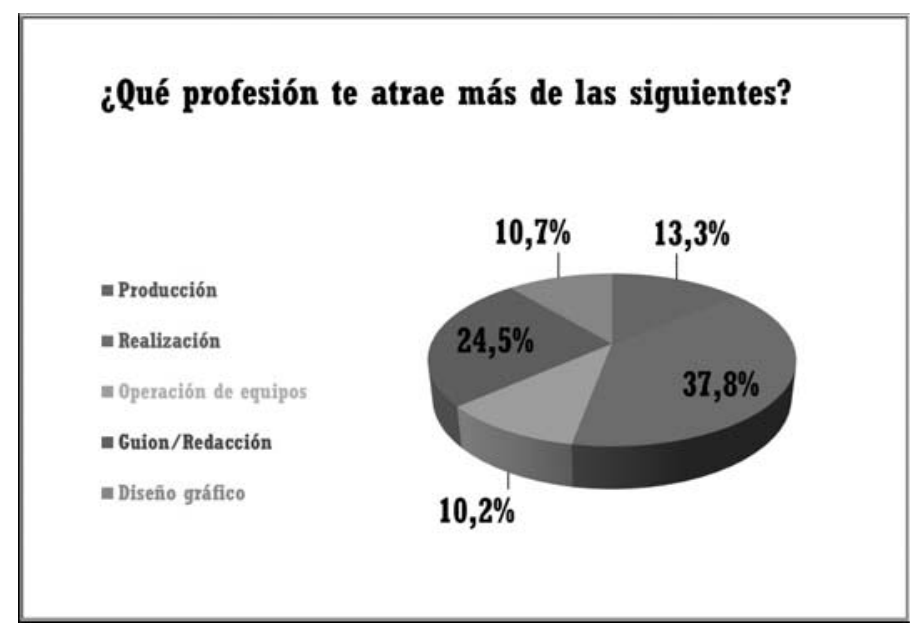

Gráfico 2. Pregunta 8. Fuente: elaboración propia.

Puede intuirse que los estudiantes valoran el conocimiento tecnológico y aspiran a manejar con solvencia los equipos de producción, si bien entre sus intereses profesionales no es mayoritaria la opción de aspirar a convertirse en operadores: solo un $10 \%$ admite esta posibilidad frente a un $25 \%$ que se decanta por actividades relacionadas con el guion y la redacción o el 50\% que se inclina hacia profesiones del ámbito de producción y realización

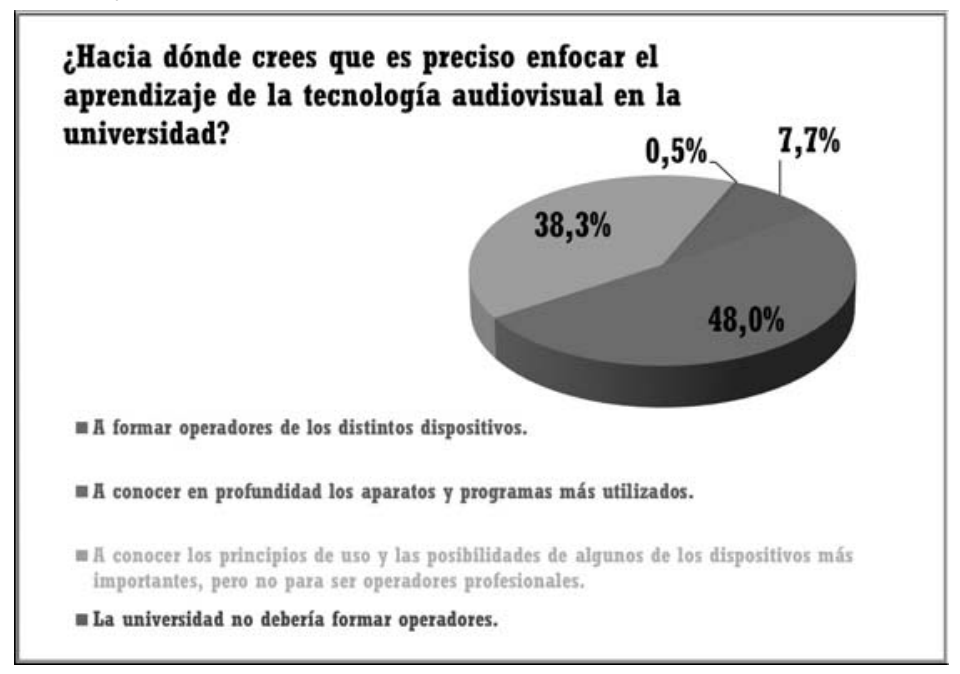

Gráfico 3. Pregunta 12. Fuente: elaboración propia. 


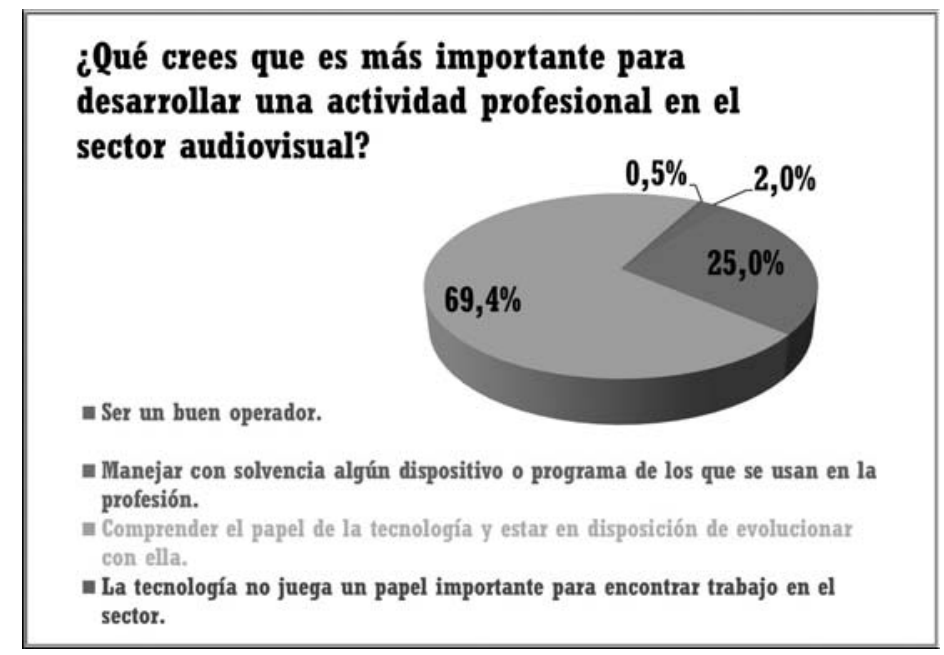

Gráfico 4. Pregunta 13. Fuente: elaboración propia.

Mayoritariamente aspiran a comprender el valor de la tecnología y estar en condiciones de evolucionar con ella y en gran medida esperan que sea la Universidad quien les suministre esos conocimientos.

\section{En relación con las clases presenciales y los procedimientos de autoaprendizaje...}

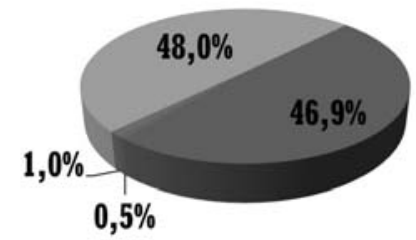

= Este grado podría cursarse completamente en forma no presencial.

= Algunos conocimientos de este grado, como los relativos a la tecnología audiovisual, podrian cursarse en forma no presencial.

as imposible cursar la tecnología audiovisual en forma no presencial, porque exige trabajo en equipo.

Es posible la creación de un sistema de aprendizaje mixto, con actividades presenciales y no presenciales en relación a la tecnología audiovisual.

Gráfico 5. Pregunta 15. Fuente: elaboración propia.

Resulta concluyente comprobar que apenas un $2 \%$ considera posible seguir el programa docente de este tipo de asignaturas en forma no presencial. ${ }^{3}$

${ }^{3}$ En la distribución de porcentajes presentados en los gráficos no se incluye el que corresponde a la opción NS/NC. 
Los resultados cualitativos de la experiencia han sido dispares aunque en general alentadores. Aproximadamente un $25 \%$ de los vídeos producidos han alcanzado, a juicio de los profesores, una alta calidad formal, mientras que un $14 \%$ presentan defectos notables de operación -exposición inadecuada, defectos de sonido, problemas de edición...- o errores de construcción del discurso audiovisual, como problemas de continuidad o planificaciones inadecuadas - encuadres ineficaces o movimientos de cámara toscos e inapropiados-.

En cuanto al desarrollo de los contenidos, los resultados obtenidos son muy similares. Y son excepcionales los casos en los que una excelente factura formal no explica convenientemente el concepto elegido o, al contrario, una realización deficiente trunca un guion impecable.

Más del $80 \%$ de los vídeos didácticos entregados se consideraron aptos para que, en una tercera fase del proyecto, fuesen publicados y puestos a disposición del alumnado como recurso de consulta.

Se cierra así un círculo en el que los propios alumnos, en un entorno de aprendizaje colaborativo, alcanzan los objetivos docentes de ciertas asignaturas de su área académica, produciendo los recursos necesarios para dotarse, al mismo tiempo y de forma autónoma, de la capacidad tecnológica y los conocimientos previos que sus estudios presuponen.

Cabe poner de manifiesto que la utilización sistemática de las TIC y los vídeos didácticos en el área de Periodismo y Comunicación Audiovisual no constituye un mero recurso de apoyo, como sucede en otras disciplinas académicas, sino que la correcta elaboración de los mismos puede también constituir, y de hecho constituye, un objetivo prioritario de algunas asignaturas del área como puede ser el caso de aquellas relacionadas con el guion la realización y la producción.

\section{Conclusiones}

A pesar de que las recomendaciones de la declaración de Bolonia para la puesta en marcha del EEES sugieren la necesaria adaptación del alumno al mercado de trabajo, los planes de estudio actuales y la distribución de la docencia no permiten dedicar, en enseñanza presencial y en las actuales circunstancias, el tiempo necesario para resolver estos retos.

Los alumnos parecen tener la percepción de que su capacitación tecnológica será esencial en su futuro laboral y, aunque no consideran que en sí misma deba constituir el objetivo final de su educación superior, sí demandan que la Universidad les ofrezca esta formación complementaria.

Aprovechando las nuevas utilidades de las propias tecnologías de la información es posible crear entornos colaborativos que permitan al estudiante capacitarse tecnológicamente sin que ello suponga una disminución apreciable del tiempo dedicado a los contenidos centrales de las asignaturas implicadas o una degradación de los objetivos docentes perseguidos.

La experiencia realizada ha consistido en la producción de videos didácticos de corta duración realizados por el propio alumnado distribuido en grupos de trabajo, con el fin de que pudiesen ser utilizados como recurso didáctico. En conjunto, ha re- 
sultado satisfactoria, abriendo así una vía alternativa para afrontar el problema de adaptación a la tecnología audiovisual como herramienta.

Cierto porcentaje de vídeos que no ha alcanzado el nivel mínimo previsto ha revelado deficiencias en el guion que ponían en duda la validez teórica de las explicaciones aportadas y consecuentemente su capacidad como recurso de autoaprendizaje. Esto indica que es absolutamente ineludible que el profesor ejerza una labor supervisora y de guía en este entorno de aprendizaje colaborativo.

En resumen, en ausencia de datos válidos de inserción laboral de egresados de ambas áreas, se evidencia que existen fórmulas de enseñanza-aprendizaje que pueden aplicarse a estos problemas tecnológicos complejos, desarrollando al mismo tiempo competencias genéricas como el trabajo en equipo, la responsabilidad y la motivación frente a la propia formación.

\section{Referencias bibiográficas}

AEBLI, Hans (2001): Factores de la enseñanza que favorecen el aprendizaje autónomo. Madrid, Narcea.

ANECA (2005): Libro Blanco de los títulos de Grado en Ciencias de la Comunicación. http/www.es/var/media/150336/libroblanco_comunicacion_def.pdf. [fecha de consulta: 10 de julio de 2012].

APARICIO IZQUIERDO, Francisco (1985): “Diseño, producción y experimentación de un curso audiovisual en vídeo para la enseñanza de la ingeniería de los vehículos automóviles". http://www.doredin.mec.es/documentos/008198600245.pdf. [fecha de consulta: 28 de junio de 2012].

BOUD, David (2001): "Conclusions: challenges and new directions" en BOUD, David, COHEN, Ruth y SAMPSON, Jane (edits): Peer Learning In Higher Education. Londres, Kogan Page, pp. 170-177.

BRAVO RAMOS, Juan Luis (2000): "E1 vídeo educativo". http://www.ice.upm.es /wps/jlbr/Documentacion/Libros/Videdu. [fecha de consulta: 28 de junio de 2012].

CABERO ALMENARA, Julio y MÁRQUEZ FERNÁNDEZ, Dominga et al. (Dirs., 2002): "La introducción del vídeo como instrumento de conocimiento en la enseñanza universitaria". http://tecnologiaedu.us.es/cuestionario/bibliovir/122.pdf. [fecha de consulta: 29 de junio de 2012].

CARRÉ, Philippe (2006): "Portée et limites de l'autoformation dans une culture de l'apprenance", http://archives.enfa.fr/autoformation/rub-pres/pcarre.pdf.[fecha de consulta: 18 de septiembre de 2011].

CENICH, Gabriela y SANTOS, Graciela (2005): "Propuesta de aprendizaje basado en proyectos y trabajo colaborativo: experiencia de un curso en línea", en Revista Electrónica de Investigación Educativa, Vol 7, nº. 2. http://redie.uabc.mx/vol7no2 /contenido-cenich.html. [fecha de consulta: 12 de junio de 2012].

DÍAZ TREMARÍAS, Marisela y NORIEGA VELÁSQUEZ, Teresa (2009): "Utilización de videos didácticos como innovación en la enseñanza de la toxicología", en 
Revista Educación Médica Superior, vol.23, n.3. http://scielo.sld.cu/scielo.php ?script=sci_arttext\&pid=S0864-21412009000300004\&lng=es\&nrm=iso. [fecha de consulta: 29 de junio de 2012].

JIMÉNEZ MARTÍN, Silvia y MONCHOLI, Miguel Ángel (2009): “La adecuación de la docencia de radio y televisión a los postulados de Bolonia”, en Icono 14, $\mathrm{n}^{\circ} .14$, pp. 139-148.

KANT, Inmanuel (1803): Tratado de pedagogía. Composición y traducción de Lorenzo Luzuriaga. Edición digital: Alicante: Biblioteca Virtual Miguel de Cervantes, 1999. http://bib.cervantesvirtual.com/servlet/SirveObras/024374082118044 95222202/index.htm

RUÉ, Joan (2009): El Aprendizaje Autónomo en Educación Superior. Madrid, Narcea. RUIZ DÁVILA, María (coord., 2004): Las TIC, un reto para nuevos aprendizajes: Usar información, comunicarse y utilizar recursos. Madrid, MEC/ Narcea.

SÁNCHEZ, Manuel, PUEO, Basilio y BENÍTEZ, Anto J. (2011). “Tecnología y pensamiento: un vínculo fundamental en la enseñanza de las tecnologías de los medios audiovisuales", en SIERRA, Javier y LIBERAL, Sheila (coords.): Investigaciones educomunicativas en la sociedad multipantalla. Madrid, Fragua, Biblioteca de Ciencias de la Comunicación, pp. 659-676.

SINGHAL, Arvind y BROWN, William J. (1996): "The Entertainment-Education Communication Strategy: Past Struggles, Present Status, Future Agenda", en Jurnal Komunikasi. Vol. 12. http://www.ukm.my/jkom/journal/pdf_files/1996 /V12_2.pdf. [fecha de consulta: 16 de septiembre de 2011].

WANG, Charles. X. y KINUTHIA, Wanjira (2004): "Defining technology enhanced learning environments for pre-service teachers", en Proceedings of Society for Information Technology and Teacher Education International Conference, 2004 (1), 2724-2727. http://www.editlib.org/?fuseaction=Reader.PrintAbstract\&paper_id $=13252$ [fecha de consulta: 15 de septiembre de 2012].

WOOD, E. J. (2004): "Problem-based learning: Exploiting knowledge of how people learn to promote effective learning". http://www.bioscience.heacademy.ac.uk/journal/vol3/beej-3-5.pdf. [fecha de consulta: 15 de septiembre de 2011].

Agradecimiento: Esta investigación se ha desarrollado en el contexto del proyecto aprobado en la $9^{\mathrm{a}}$ Convocatoria de Apoyo a Experiencia de Innovación e Internacionalización Docente (curso 2011/2012) de la Universidad Carlos III de Madrid. Los autores quieren significar y agradecer el trabajo y la participación en dicho proyecto de los profesores Manuel Armenteros, Manuel Caballero, José Cuevas, David López Buforn, Jesús González, Bruno López Pacheco, Luis Serrano, Francisco Utray y Antonio Weinrichter. 


\section{Antonio J. BENÍTEZ IGLESIAS}

abenitez@hum.uc3m.es

Edificio Ortega y Gasset; despacho 17.2.71; C/ Madrid, 133. 28903 Getafe - Madrid Universidad Carlos III de Madrid

Doctor en Comunicación Audiovisual

\section{Esteban M. STEPANIAN TARACIDO}

estepani@hum.uc3m.es

Edificio Ortega y Gasset; despacho 17.2.39; C/ Madrid, 133. 28903 Getafe - Madrid Universidad Carlos III

Doctor en Ciencias de la Información. Realizador de televisión y profesor de Tecnología de los Medios Audiovisuales y Teoría y Técnica de la Realización Audiovisual. 